Artículo de investigación.

Cómo citar: K. González, L. Pérez, y E. Galeano, "Análisis del ciclo de vida de un edificio residencial en Colombia", Inventum, vol. 14 , no. 27 , pp. 3-14. doi: 10.26620/ uniminuto.inventum.14.27.2019.3-14

Editorial: Corporación Universitaria Minuto de Dios - UNIMINUTO.

ISSN: $1909-2520$

eISSN: 2590-8219

Fecha de recibido: mayo 01 de 2019 Fecha de aprobado: junio 30 de 2019 Fecha de publicación: septiembre 01 de 2019

Conflicto de intereses: los autores han declarado que no existen intereses en competencia.

\section{Análisis del ciclo de vida de un edificio residencial en Colombia}

\author{
Analysis of the life cycle of \\ a residential building in Colombia
}

\author{
Análise do ciclo de vida de \\ um edifício residencial na Colômbia
}

Krystle Danitza González Velandia http://orcid.org/0000-0002-6982-2569 kgonzalez@uniminuto.edu Corporación Universitaria Minuto de Dios UNIMINUTO, Colombia.

Luisa Fernanda Pérez http://orcid.org/0000-0002-8821-3902 Ipereznavar@uniminuto.edu.co Corporación Universitaria Minuto de Dios UNIMINUTO, Colombia.

Erika Galeano http://orcid.org/0000-0003-0275-3033 egaleanomar@uniminuto.edu.co Corporación Universitaria Minuto de Dios . UNIMINUTO, Colombia.

\begin{abstract}
Resumen
A nivel mundial se han adelantado diferentes estudios que revelan los impactos ambientales del sector de la construcción. Estos impactos se asocian a los materiales, la maquinaria empleada, las técnicas que se aliquen, a las condiciones geográficas donde se ubique la construcción, y la demanda de energía y recursos durante su uso. En el caso colombiano, son escasos los estudios de impactos ambientales en el sector de la construcción, y aún más escasos en construcciones verticales, lo que motivó la presente investigación centrada en la evaluación de los impactos ambientales de un edificio residencial de doce pisos en Bogotá, D. C., empleando un análisis de ciclo de vida. Este análisis se realiza desde la fase de producción de los materiales de construcción, transporte, la construcción, el uso y hasta su disposición final, aplicando el método de evaluación de impacto CML- 2015. Los resultados muestran que entre los principales impactos están la $A D$ y el GWP, y que en la etapa de uso es donde se presentan el $97 \%$ del total de los impactos ambientales del edificio, por ser la etapa donde más se consume energía y agua, especialmente asociado a hábitos de consumo.
\end{abstract}

Palabras clave: construcción de edificios, energía incorporada, análisis de ciclo de vida, impactos ambientales, calentamiento global, desarrollo sostenible. 
the main impacts are AD and GWP, and that the use stage is where $97 \%$ of the total environmental impacts of the building are present, as it is the stage where most energy and water is consumed, especially associated with consumption habits.

Keywords: building construction, incorporated energy, life cycle analysis, environmental impacts, global warming, sustainable development.

\section{Resumo}

Diferentes estudos têm sido realizados em todo o mundo que revelam os impactos ambientais do sector da construção. Estes impactos estão associados aos materiais, às máquinas utilizadas, às técnicas utilizadas, às condições geográficas em que a construção está localizada e à procura de energia e recursos durante a sua utilização. No caso da Colômbia, os estudos de impacto ambiental no setor da construção são escassos, e ainda mais escassos em construções verticais, o que motivou a presente pesquisa focada na avaliação dos impactos ambientais de um edifício residencial de doze andares em Bogotá, D. C., utilizando uma análise do ciclo de vida. Esta análise é realizada desde a fase de produção dos materiais de construção, transporte, construção, utilização e disposição final, aplicando o método de avaliação de impacto CML- 2015. Os resultados mostram que entre os principais impactos ocorrem durante o AD e o GWP, e que na fase de uso é onde são apresentados $97 \%$ dos impactos ambientais totais do edifício, pois é a fase em que mais energia e água são consumidas, especialmente associada a hábitos de consumo.

Palavras-chave: construção de edifícios, energia incorporada, análise do ciclo de vida, impactos ambientais, aquecimento global, desenvolvimento sustentável.

\section{Glosario:}

ACV: análisis de ciclo de vida / life cycle assessment

GWP: calentamiento global potencial /.global warming potential

AP: acidificación potencial / acidification potential

EP: eutrofización potencial / eutrophication potential

HT: toxicidad humana / human toxicity

FAETP: ecotoxicidad en agua dulce / fresh water aquatic ecotoxicity MAETP: ecotoxicidad acuática marina / marine aquatic ecotoxicity TETP: ecotoxicidad terrestre / terrestrial ecotoxicity OD: agotamiento de la capa de ozono/ ozone layer depletion AD: agotamiento de recursos abióticos / abiotic depletion 


\section{INTRODUCCIÓN}

En Colombia se ha registrado un incremento en la construcción de vivienda vertical, asociado a la necesidad de optimizar áreas. El 86,2\% de las viviendas que se desarrollan en el país son apartamentos, y para el caso de Bogotá, D. C., la cifra se acerca al $70 \%$ [1]. Al lado de este auge en el sector de la construcción, se están generando una serie de impactos negativos en el ambiente, como lo muestran algunos estudios donde indican que este sector es responsable a nivel global de la extracción del 24 $\%$ de minerales de la litosfera [2], sumado a la demanda de energía para el transporte de materiales, producción y equipos de construcción [3], [4].

A nivel mundial se han adelantado diferentes estudios que revelan los impactos del sector de la construcción. Por ejemplo, la producción de materiales como ladrillos genera una cantidad importante de $\mathrm{CO}_{2}$ a la atmosfera [5]; y la explotación de canteras para la obtención de materias primas, que aparte de estar en disputa su legalidad en algunas zonas, impacta negativamente el medio ambiente con la generación de gases por combustión no controlada y la alteración de los ecosistemas [6]. El tipo de material, el proceso de extracción, la tecnología de producción, las distancias de transporte hasta el sitio de obra y el tipo de estructura influyen directamente en el nivel de impacto, siendo los más frecuentes la GWP y el AD, [7], [8], [9], [10], [4].

Los impactos de una construcción, en este caso de un edificio, obedecen a condiciones locales. Los análisis ambientales basados en los análisis de ciclo de vida (ACV), en los que se examina todo el proceso desde la extracción de materiales hasta la disposición final, desarrollados en el sector.de la construcción en Colombia, son limitados, pero particularmente para el caso colombiano hay un estudio desarrollado por Ortiz, Castells y Sonnemann [11], donde se comparó el ACV de una casa ubicada en el municipio de Pamplona (Colombia) con respecto a una casa en Barcelona (España), en donde encontró que el mayor impacto ambiental se da en la etapa del uso de la vivienda para ambos casos, pero es mayor en Barcelona por la demanda energética en periodos de invierno y verano, es decir por las particularidades de su condición geográfica [12]. Por tal motivo, esta investigación presenta un estudio de caso centrado en el análisis de ciclo de vida de un edificio residencial de 13 pisos ubicado en Bogotá, D. C., con la intención de identificar aquellas etapas y procesos más impactantes en el contexto colombiano, y que sirva como base para futuras investigaciones.
Estudios de análisis de ciclo de vida en la construcción

La evaluación de impactos con el ACV en el sector de la construcción ha sido ampliamente usada en todo el mundo, debido a la tendencia del sector de volverse sostenible. Los estudios se han centrado en la evaluación de las diferentes etapas implicadas en la construcción, sus correspondientes impactos y el análisis de materiales empleados. La producción del cemento genera impactos como el agotamiento de recursos [13], [14], [15], pero la manufactura y transporte de este material genera grandes emisiones de $\mathrm{CO}_{2}$, por tener una alta demanda de energía fósil, [15], [16], [17], [13], resultados que coinciden con estudios realizados en hormigón [18] y en áridos [19].

Otro factor determinante en la generación de impactos es la tecnología que se emplee.[20] comparó la producción de ladrillos artesanales, ladrillos mecanizados y bloques de concreto producidos, y encontró que un ladrillo mecanizado impacta al ambiente en un $36 \%$ más de emisiones de $\mathrm{CO}_{2}$ - eq que la obtención de un ladrillo artesanal, y un bloque de concreto impacta en un $175 \%$ y $102 \%$ más que la producción de un ladrillo artesanal y un ladrillo mecanizado, respectivamente, asociado al consumo de energía y al transporte de materiales. Estos resultados coinciden con los de un estudio realizado en la universidad de Arizona, donde se efectuó el análisis a la fabricación de ladrillos a partir de materiales de desecho, allí se encontró que los ladrillos de arcilla, en promedio, tienen una energía incorporada de aproximadamente 2,0 kWh y liberan aproximadamente $0.41 \mathrm{~kg}$ de dióxido de carbono $\left(\mathrm{CO}_{2}\right)$ por ladrillo [21].

El ACV también se ha empleado para analizar y comparar los impactos ambientales generados por diferentes tipos de construcciones como viviendas, oficinas, y otros, en diferentes partes del mundo. La unidad de analisis de estos estudios ha sido el metro cuadrado, o su equivalente en pies cuadrados [22], [11], [23], [24], [25], [4], [10], [26], [27], [28].

Los ACV han señalado que la fase de construcción tiene un impacto que oscila entre el $10 \%$ al $23 \%$ del total, debido principalmente a la cadena de suministro de materiales [25], [28], [23], [29], [30], [7], [8]. El uso de la estructura representa la etapa de mayor impacto, entre un $70 \%$ y un $90 \%$, causado. por el consumo de electricidad utilizado en sistemas de refrigeración, calefacción e iluminación [29], [23],.[10], y la fase de fin de vida y disposición final contribuye entre el $1 \%$ al $9 \%$ de los impactos totales 
[25], [11], [31]. Estos resultados muestran la utilidad que puede tener un ACV en la implementación de nuevos materiales y en mejorar en los procesos de producción y construcción.

Debería incluirse información sobre el método de evaluación CML-2015 y sobre el software OpenLCA (software libre para análisis de ciclo de vida ${ }^{4}$ ) para contextualizar al lector. También algo breve de la norma ISO 14040.

\section{METODOLOGÍA}

La presente investigación consistió en un caso de estudio que documenta el ACV de un edificio en Bogotá, D. C., en Colombia, siguiendo las directrices de la ISO 14040 (análisis del ciclo de vida, principios y marco de referencia) [32]. El análisis se realizó a un edificio de 13 pisos y 2 sótanos, con un área de 524,4 $\mathrm{m}^{2}$ ubicado en la ciudad de Bogotá, ciudad a una altura de 2600msnm, un clima bimodal (por la ubicación del país en una zona tropical no se presentan estaciones), moderadamente frío, con temperaturas promedio de $14{ }^{\circ} \mathrm{C}$, y humedad relativa cercana al $80 \%$. Estas características climáticas no demandan el uso de electrodomésticos ni energía para el calentamiento o enfriamiento en las construcciones, que es lo habitual en la ciudad. El alcance del análisis del estudio abarcó desde la extracción de materiales hasta la disposición final, contemplando la producción de los materiales de construcción, transporte, el ensamblaje de los mismos, consumo de electricidad, agua potable y gas natural, hasta el final de vida y la disposición final de los componentes, organizadas en 3 fases como se aprecia en la figura 1.

Figura 1. Diagrama de flujo de las fases de producción de un edificio residencial

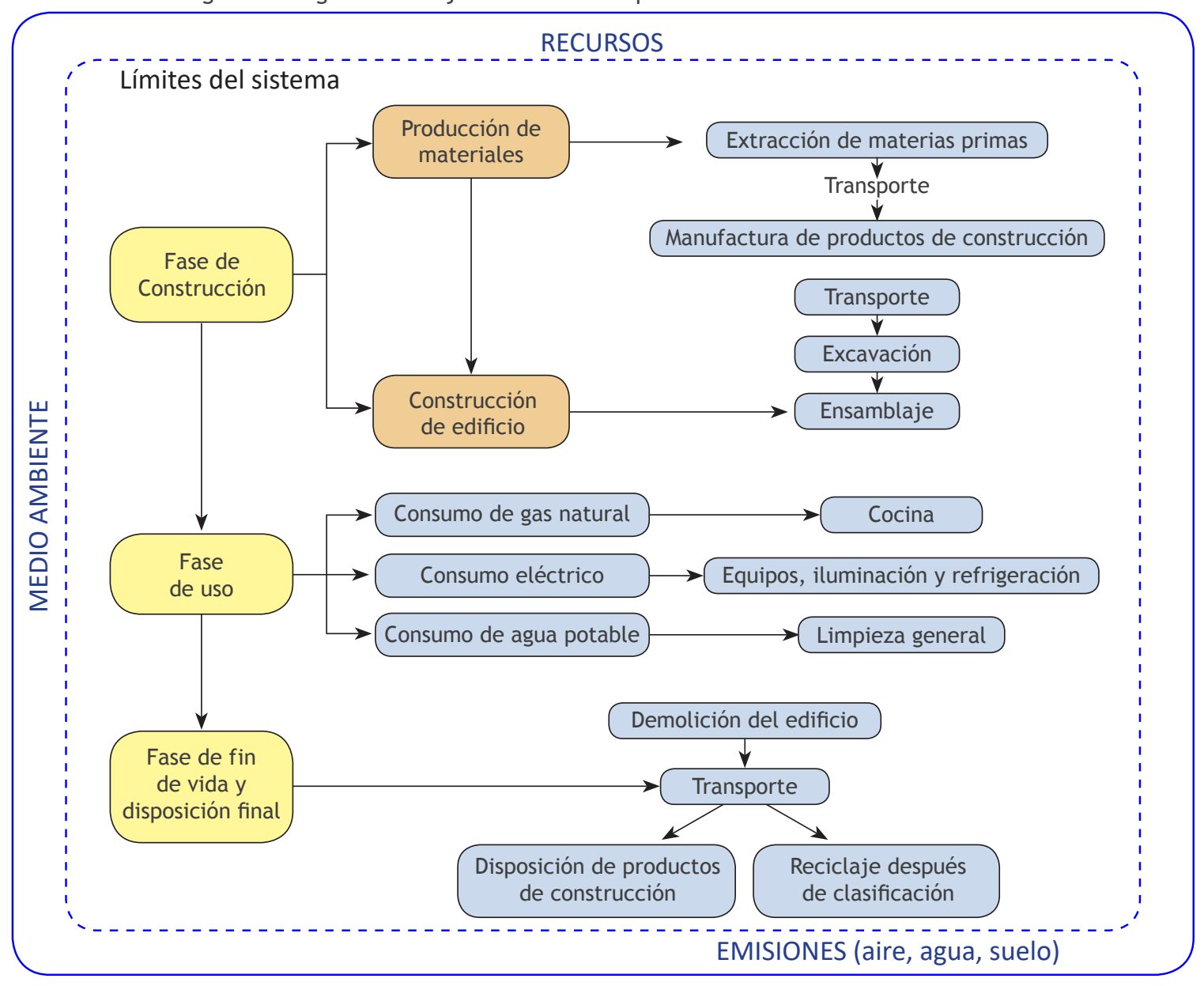

Fuente: elaboración propia.

4 http://www.openlca.org/ 
La información primaria, es decir, materiales, cantidades, equipos, tiempos de uso y consumos de energía, se recolectaron y evaluaron directamente en la construcción durante un periodo de seis meses. Para las etapas posteriores a la construcción se utilizaron fuentes secundarias como consumos específicos de empresas que ofrecen servicios públicos en Colombia como la Empresas Públicas de Medellín EPM [33], y una revisión del manejo de residuos en Bogotá, D. C. Para la información de impactos que se generan en la producción de cada material empleado en la construcción, la fuente de datos fue la base de datos Ecoinvent 3.35. [34], con la que se ajustó la cadena de suministro de todos los insumos seleccionados, considerando la ubicación de la construcción, los medios de transporte y las distancias recorridas, para aumentar la confiabilidad. El inventario en cada fase se ajustó a la unidad funcional y su vida útil, en este caso, un metro cuadrado $\left(\mathrm{m}^{2}\right)$ y 50 años de vida útil. Se consideraron las entradas y salidas insumos, maquinaria, residuos, entre otros, en términos de masa (Kilogramos) y de energía para cada una de las fases establecidas reflejadas en la figura 1 [25], [35], [36], [37].

En la fase de construcción se consideraron las cantidades de materiales empleados, equipos y el consumo de energía asociado la distribución de estos materiales desde el lugar de producción hasta el sitio de construcción de los edificios. Se estimó el peso total de todos los elementos de construcción y se encontraron las distancias desde las plantas de producción hasta el sitio de la obra. También se definieron los equipos y maquinaria empleados en el proceso constructivo del edificio, contabilizando de igual manera el consumo de combustible referente al rendimiento y tiempo de uso. Toda esta información se estableció a partir del manejo de la construcción, es decir que los datos de uso, de tiempo y cantidades se estimaron directamente en la obra.

En la fase de uso se estimaron todas las actividades relacionadas con el uso de una vivienda, como el consumo de agua, la electricidad y el gas natural. El agua que se utiliza para todo el proceso de abastecimiento y limpieza en el hogar, la electricidad es empleada para los diferentes dispositivos electrónicos y la iluminación de los hogares y el gas natural es usado para la producción de agua caliente y la cocina.

En la fase de fin de vida se consideraron tres opciones para la eliminación del material de construcción: reciclaje directo, reciclado parcial después de la clasificación y eliminación sin reciclado. La resolución 2397 de 2011 de la Secretaría Distrital de Ambiente exige para el 2017 la recuperación 25 $\%$ del total de metros cuadrados del proyecto en componentes no estructurales de los residuos generados por las etapas constructivas y de desmantelamiento. Con el fin de cumplir los requisitos de la norma, se asume que el acero se recicla en un 100 $\%$, y que el $25 \%$ de los escombros de la demolición del edificio (i.e. concreto, ladrillos, mortero, pvc) se reciclan parcialmente en los centros de tratamiento y/o aprovechamiento legalmente constituidos, siendo el restante $(75 \%)$ enviado a los sitios de disposición final.

Tabla 1. Datos del inventario de ciclo de vida

\begin{tabular}{|l|l|r|}
\hline \multicolumn{1}{|c|}{ Fase } & \multicolumn{1}{c|}{ Material } & \multicolumn{1}{c|}{ Cantidad } \\
\hline & Concreto & $4.071 \mathrm{~m}^{3}$ \\
\hline & Acero & $271.508 \mathrm{Kg}$ \\
\hline & Ladrillo & $56.481 \mathrm{UN}$ \\
\hline Producción & Mortero & $115 \mathrm{~m}^{3}$ \\
\hline y transporte & Tubería agua potable PVC & $2.123 \mathrm{Kg}$ \\
\hline & Tubería alcantarillado PVC & $3568 \mathrm{Kg}$ \\
\hline & Tubería gas & $2.265 \mathrm{Kg}$ \\
\hline & Combustible & $1.071,02 \mathrm{Gal}$ \\
\hline
\end{tabular}

Fuente: elaboración propia.

5 Centro Suizo para Inventarios del Ciclo de Vida. Base de datos de inventario de ciclo de vida. www.ecoinvent.org 


\begin{tabular}{|c|c|c|}
\hline Fase & Material & Cantidad \\
\hline \multirow{7}{*}{ Construcción } & $\begin{array}{l}\text { Combustible } \\
\text { Retroexcavadora Oruga - Komatsu PC } 200\end{array}$ & $2.796,38 \mathrm{Gal}$ \\
\hline & $\begin{array}{l}\text { Combustible } \\
\text { Retroexcavadora Oruga - Bobcat E42 }\end{array}$ & $379,80 \mathrm{Gal}$ \\
\hline & $\begin{array}{l}\text { Combustible } \\
\text { Minicargador }\end{array}$ & $633,00 \mathrm{Gal}$ \\
\hline & $\begin{array}{l}\text { Combustible } \\
\text { Volquetas doble troque }\end{array}$ & $1.922,94 \mathrm{Gal}$ \\
\hline & $\begin{array}{l}\text { Combustible } \\
\text { Hincadora - } 240 \text { Ton de capadidad }\end{array}$ & $263,18 \mathrm{Gal}$ \\
\hline & $\begin{array}{l}\text { Combustible } \\
\text { Hincadora - } 240 \text { Ton de capadidad }\end{array}$ & $1,99 \mathrm{Gal}$ \\
\hline & $\begin{array}{l}\text { Combustible } \\
\text { Bomba Pultzmeister tk } 70\end{array}$ & $1.221,39 \mathrm{Gal}$ \\
\hline \multirow{3}{*}{ Uso } & Consumo agua & $442.800 .000 \mathrm{~m}^{3}$ \\
\hline & Consumo electricidad & $21.600 .000 \mathrm{kWh}$ \\
\hline & Consumo gas natural & $518.400 \mathrm{~m}^{3}$ \\
\hline \multirow{2}{*}{ Fin de vida } & Transporte de material de desecho y reciclable & $8.838 .660 \mathrm{Kg}$ \\
\hline & Transporte material de excavación & $15.352 .216 \mathrm{Kg}$ \\
\hline
\end{tabular}

Fuente: Elaboración propia.

Una vez listo el inventario se procedió a la evaluación de impactos a partir del ACV. En el presente caso, se utilizó la metodología de CML - 2015 [38] para la caracterización de 11 categorías de impacto: agotamiento de ozono OD, acidificación potencial AP, oxidación fotoquímica, ecotoxicidad en agua dulce FAETP, ecotoxicidad acuática marina MAETP, calentamiento global potencial GWP, ecotoxicidad terrestre TETP, eutrofización potencial EP, toxicidad humana HT, agotamiento de recursos abióticos $A D$.

Este análisis se realizó con el apoyo del software OpenLCA 1.6.3 [39], el cual es libre y de código abierto para la evaluación del ciclo de vida y sostenibilidad y la base de datos Ecoinvent 3.3, la cual es un inventario del ciclo de vida de productos de agricultura, transporte y construcción [34].

El análisis contempló evaluar los impactos etapa por etapa y además hacer un análisis global de los principales impactos causados por el edificio en Bogotá, Colombia.

\section{RESULTADOS Y DISCUSIÓN}

A partir del ACV se obtuvieron los impactos generados por el edificio, como se aprecia en la tabla 2. Entre los impactos presentados está el AD con 1,85E+ $07 \mathrm{MJ}\left(3,52 \mathrm{E}+04 \mathrm{MJ} \mathrm{m}^{-2}\right)$ con mayor presencia en la fase de uso. Otro importante impacto es el GWP con 8,22E+ $05 \mathrm{~kg} \mathrm{CO}$ $\left(1,57 \mathrm{E}+03 \mathrm{~kg} \mathrm{CO}\right.$ eq $\left.\mathrm{m}^{-2}\right)$ presente en todas las etapas de análisis, pero más alto en la fase de uso, la MAETP con 1,12E+ $04 \mathrm{~kg} 1,4-\mathrm{DB}$ eq $(2,13 \mathrm{E}+01 \mathrm{~kg}$ 1,4-DB eq $\mathrm{m}^{-2}$ ) presente en la fase de extracción de materiales, la HTTP con 9, 99E + $03 \mathrm{~kg} 1,4$-DB eq $(1,90 \mathrm{E}$ $+01 \mathrm{~kg} 1,4-\mathrm{DB}$ eq $\mathrm{m}-2$ ) presente en la fase de construcción y el AP con $1,66 \mathrm{E}+03 \mathrm{~kg} \mathrm{SO} 2$ eq $(3,17 \mathrm{E}+00 \mathrm{~kg}$ $\mathrm{SO}_{2}$ eq $\mathrm{m}-2$ ) generado principalmente fase de uso.

La fase de uso fue la de mayor impacto, como se puede apreciar en la figura 2. En esta etapa se genera el $97 \%$ del total de los impactos ambientales, debido a que la vida útil del edificio evaluada fue de 50 años, y durante el uso fue donde más se empleó energía y agua. 
Tabla 2. Impactos ambientales del análisis de ciclo de vida por fase

\begin{tabular}{|c|c|c|c|c|c|c|}
\hline Fase & Elemento o maquinaria & $\begin{array}{c}\mathrm{AP} \\
\left(\mathrm{kg} \mathrm{SO}{ }_{2} \text { eq) }\right.\end{array}$ & $\begin{array}{c}\text { GWP } \\
\text { (kg CO}{ }_{2} \text { eq) }\end{array}$ & $\begin{array}{c}\text { AD } \\
\text { (kg Sb eq) }\end{array}$ & $\begin{array}{c}\text { HTTP } \\
\text { (kg 1,4-DB eq) }\end{array}$ & $\begin{array}{c}\text { MAETP } \\
\text { (kg 1,4-DB eq) }\end{array}$ \\
\hline \multirow{7}{*}{ Extracción } & Concreto & $3,74 \mathrm{E}+01$ & $2,39 \mathrm{E}+03$ & $5,54 \mathrm{E}+03$ & $2,24 \mathrm{E}+03$ & $3,31 \mathrm{E}+03$ \\
\hline & Acero de refuerzo & $1,03 E+00$ & $1,39 \mathrm{E}+04$ & - & $3,18 \mathrm{E}+01$ & $1,61 \mathrm{E}+02$ \\
\hline & Ladrillo de arcilla & $3,67 \mathrm{E}+01$ & $2,65 E+04$ & - & $8,75 E+02$ & $1,18 \mathrm{E}-02$ \\
\hline & Mortero & - & - & - & - & - \\
\hline & Policloruro de vinilo & $2,86 \mathrm{E}+01$ & $1,07 E+04$ & $2,46 E+05$ & $2,43 E+02$ & $7,01 \mathrm{E}+02$ \\
\hline & Madera contrachapada & - & - & - & $5,36 \mathrm{E}-01$ & $1,05 \mathrm{E}+00$ \\
\hline & Transporte $16-32$ Ton & $3,14 \mathrm{E}+01$ & $7,89 \mathrm{E}+03$ & - & $8,69 \mathrm{E}+01$ & $4,96 \mathrm{E}+02$ \\
\hline \multirow{3}{*}{ Construcción } & Maquinaria < $18.64 \mathrm{~kW}$ & $5,06 \mathrm{E}+00$ & $1,24 \mathrm{E}+03$ & $1,42 E+02$ & $1,42 E+02$ & $7,21 \mathrm{E}+02$ \\
\hline & Maquinaria $>=18.64 \mathrm{~kW} \mathrm{y}<74.57 \mathrm{~kW}$ & $6,90 \mathrm{E}+01$ & $3,13 E+04$ & $1,71 \mathrm{E}+03$ & $1,71 \mathrm{E}+03$ & $1,24 \mathrm{E}+03$ \\
\hline & Maquinaria $>=74.57 \mathrm{~kW}$ & $1,68 \mathrm{E}+02$ & $8,06 \mathrm{E}+04$ & $3,19 \mathrm{E}+03$ & $3,19 \mathrm{E}+03$ & $3,42 \mathrm{E}+02$ \\
\hline \multirow{3}{*}{ Uso } & Electricidad & - & $4,66 \mathrm{E}+05$ & - & - & - \\
\hline & Gas natural & $1,03 E+03$ & $1,39 \mathrm{E}+04$ & $1,82 \mathrm{E}+07$ & $7,55 \mathrm{E}+02$ & $1,06 \mathrm{E}+02$ \\
\hline & Agua potable & - & $1,02 E+05$ & - & - & - \\
\hline \multirow{8}{*}{$\begin{array}{l}\text { Fin de vida } \\
\text { y disposición } \\
\text { final }\end{array}$} & Producción de nitrato de amonio & 2,97E-03 & - & - & $2,47 \mathrm{E}-03$ & - \\
\hline & Residuos de concreto & - & - & - & - & - \\
\hline & Residuos de ladrillo & - & - & - & $-1,37 \mathrm{E}+01$ & - \\
\hline & Residuos de mortero & - & - & - & - & - \\
\hline & Residuos de policloruro de vinilo & - & - & - & - & - \\
\hline & Material de excavación & - & - & - & - & - \\
\hline & Transporte $16-32$ Ton & $2,59 \mathrm{E}+02$ & $6,52 E+04$ & - & $7,18 \mathrm{E}+02$ & $4,10 E+03$ \\
\hline & Total & $1,66 \mathrm{E}+03$ & $8,22 \mathrm{E}+05$ & $1,85 \mathrm{E}+07$ & $9,99 \mathrm{E}+03$ & $1,12 \mathrm{E}+04$ \\
\hline
\end{tabular}

Fuente: Elaboración propia.

Durante todo el ciclo de vida del edificio residencial, el GWP fue uno de los impactos más significativos, el mayor aporte a este impacto se presentó durante la fase de uso, representando el $71 \%$ del total de este impacto. Esto se debe a los diferentes tipos de energías no renovables que se utilizan en la producción para el abastecimiento humano, y en combustibles para el transporte de materiales o residuos [11], [40], [25].

La MAETP se presentó principalmente en la etapa de extracción con un $42 \%$ de aporte al total de este impacto durante todo el ciclo de vida, debido a que en esta fase se realiza el proceso de manufactura de materiales de obra, en donde se generan residuos líquidos sobre los cuerpos de agua, que afectan la biodiversidad acuática [41], [42]. La HTTP también se presentó en mayor proporción durante la fase de extracción y construcción con un 86 \% con respecto al total, pues en estas fases el trabajador tiene contacto con materiales de construcción - sustancias que lo pueden afectar a través de la piel, la respiración, la ingestión y el contacto con los ojos, por ejemplo, las gravas empleadas como áridos en la producción de concreto suelen ser radiactivas, estas partículas suelen descomponerse y quedar atrapadas en los pulmones [43].

Por último, el AP fue otro de los impactos ambientales generados en la fase de extracción con un $23 \%$ con respecto al total, en la fase de uso con un $62 \%$ y la fase de fin de vida del $16 \%$. Este valor, asociado a la explotación del suelo para la obtención de combustibles fósiles, genera gases contaminantes a la atmosfera (i. e. óxido de nitrógeno y dióxido de azufre), que en combinación 
Figura 2. Impactos ambientales del análisis de ciclo de vida

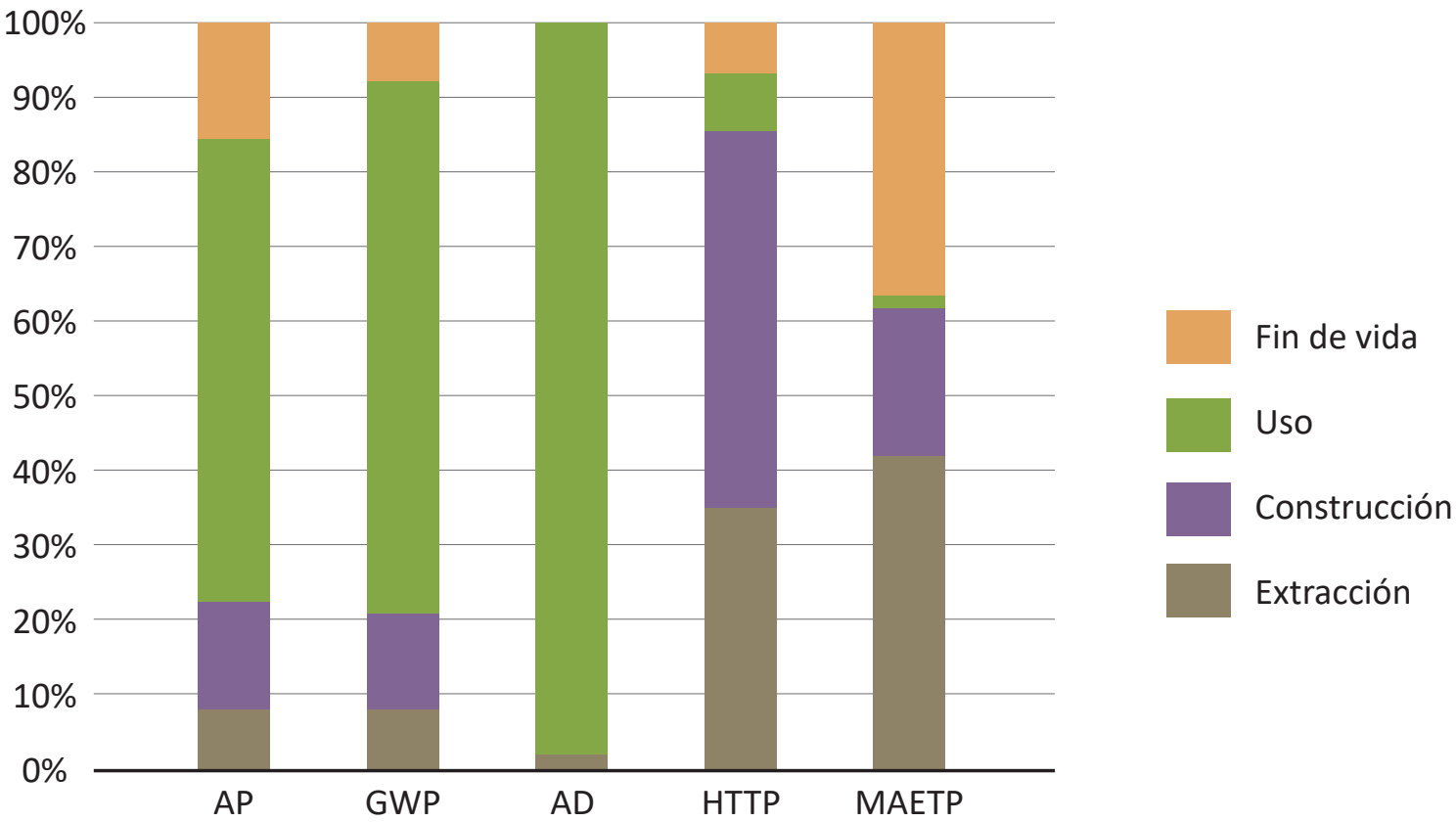

Fuente: elaboración propia.

con el oxígeno del aire y el vapor de agua se transforman en ácidos (ácido sulfúrico y ácido nítrico) para depositarse en la superficie terrestre a través de las precipitaciones [44].

Analizando lo que sucedió en cada una de las etapas, el proceso de extracción de materiales y construcción impactó con el AD en 2,51E+05 MJ, de los cuales la producción de concreto representó el $59 \%$ del total debido a la extracción de materias primas y la energía utilizada en la producción de los diferentes elementos que lo conforman [45], [46], y la fabricación del PVC que representó el 41 \% del total. El GWP está asociado a la producción del ladrillo de arcilla en un 43\%, por el uso de diversos combustibles en el proceso de cocción [47], [48], y el transporte de materiales, que representó el $13 \%$, lo que hace que en este caso no sea un gran aportante debido a que la distancia promedio desde las fábricas hasta el sitio de construcción fue en su máximo caso de $30 \mathrm{~km}$.

En la fase de construcción del edificio residencial, los impactos que más se presentaron fueron el GWP y el AD. El GWP fue el mayor impacto, generado principalmente por la maquinaria y los combustibles que estas usaban, como el diésel [49], [50] y a la antigüedad de la maquinaria utilizada actualmente en Colombia, dado que no es maquinaria de última tecnología.

La fase de uso fue la mayor contribuyente de impactos ambientales, exceptuando el OLDP [29], asociado al tiempo de vida útil del edificio. En la fase de uso el GWP estuvo asociado a la producción de electricidad, la que representó entre un $80 \%$ y $90 \%$ del total del consumo, al igual que otros estudios realizados [11], [29]. En Colombia la producción de energía se da en dos formas: a partir del aprovechamiento de la energía potencial del agua embalsada en represas, y en las plantas térmicas la energía eléctrica se obtiene a partir del poder calorífico de la combustión de elementos fósiles (gas natural, diésel, carbón). Esta combustión es la responsable de emitir $\mathrm{CO}_{2}$ [51], [52], [53]. El AD también se asocia esta situación y debido a la producción de gas natural para las viviendas, lo cual implica la extracción y manufactura de una cantidad mayor de recursos fósiles naturales [49], [50]. 
Los impactos en la fase de disposición final se dan principalmente por combustible empleado por la maquinaria para el transporte de los residuos generados por el edificio, aunque esta etapa es la que menores impactos presenta [25], [31]. En Colombia se recicla por exigencia apenas el $25 \%$, a diferencia de países europeos donde se exige para el año 2020 la recuperación del 70 \% en peso de todos los residuos [31], [54].

Comparando los resultados con un edificio en Corea del Sur, con un impacto de $3,25 \mathrm{E}+03 \mathrm{~kg} \mathrm{CO}$ eq $\mathrm{m}^{-2}$ [40] y un edificio en España, con un impacto de $2,47 \mathrm{E}+03 \mathrm{~kg} \mathrm{CO}_{2}$ eq $\mathrm{m}^{-2}[11]$, se encuentra que son mayores al impacto del edificio en Colombia $\left(1,57 \mathrm{E}+03 \mathrm{~kg} \mathrm{CO}_{2}\right.$ eq $\left.\mathrm{m}^{-2}\right)$, debido a que se emplean tecnologías diferentes, pero sobre todo a las condiciones climáticas.

Para un edificio unifamiliar en Italia de $443 \mathrm{~m}^{2}$ se obtuvo un impacto de $1,10 \mathrm{E}+03 \mathrm{~kg} \mathrm{CO}_{2}$ eq m$^{-2}$, un poco menor al edificio en Colombia debido a la disminución del área construida [25].

Este estudio y otros ACV en construcciones coinciden, además, en que potencial de calentamiento global es uno de los impactos más significativos, siendo la construcción la responsable entre $20-28 \%$, la fase de uso entre $70-92 \%$, y la fase de fin de vida entre $0-9 \%$ [11], [25], [56], [29]. Es decir, que en la fase de uso, es en la que más se presenta GWP, por lo que se debe trabajar en buscar estrategias para disminuir la emisiones que genera el consumo energético, como fuentes de energía renovables y también evitar el desperdicio de energía.

Al comparar el edificio residencial y una casa, se encuentra que mientras en el edificio, para el caso colombiano, emite aproximadamente $1,57 \mathrm{E}+03 \mathrm{~kg} \mathrm{CO}_{2}$ eq $\mathrm{m}^{-2}$ durante su ciclo de vida, la casa, según [11], emite $8,62 \mathrm{E}+02 \mathrm{~kg} \mathrm{CO}_{2}$ eq $\mathrm{m}^{-2}$, es decir, que los impactos del edificio son mayores dada la condición de espacios comunes, puesto que en el edificio tenemos áreas mayores, en especial comunes lo que devenga un mayor uso de energía para su uso y mantenimiento, generando así mayores impactos por emisiones.

\section{CONCLUSIONES}

Este estudio de caso evidencia que los impactos ambientales asociados a un edificio de trece pisos ubicado en Bogotá D.C (Colombia) están principalmente asociados a hábitos de consumo de quienes los habitan, pues la fase se uso es la que más impactos genera por la demanda de agua y energía, que a su vez está determinada por las condiciones climáticas de la estructura. En este caso, a diferencia de análisis realizados en otros sitios del mundo, son menores los impactos ambientales ya que no se demandan sistemas de calefacción o enfriamiento. Sin embargo, representa un reto para el sector de la construcción hacer que los procesos de producción de materiales sean más eficientes, pues los recursos naturales como el agua y suelo son los más impactados, a lo que se suman las emisiones que aportan al fenómeno de cambio climático asociadas al transporte y a la producción de energía.

\section{REFERENCIAS}

[1] G. Flores, "El 70 \% de la vivienda de Bogotá está construida en altura," Portafolio, 18 feb., 2017. [En línea]. Disponible en: http://www.portafolio.co/mis-finanzas/ vivienda/el-70-de-la-vivienda-debogotaesta-construida-en-altura-503480. [Último acceso: 6 Junio 2017].

[2] I. Zabalza, A. Valero y A. Aranda, "Life cycle assessment of building materials: Comparative analysis of energy and environmental impacts and evaluation of the eco-efficiency improvement potential," Building and Environment, vol. 46, n. ${ }^{\circ}$ 5, pp. 1133-1140, 2011. DOI: https://doi.org/10.1016/j.buildenv.2010.12.002.

[3] R. Lukman, A. Tiwary y A. Azapagic, "Towards greening a university campus: The case of the University of Maribor, Slovenia," Resources, Conservation and Recycling, vol.53, n. ${ }^{\circ} 11$, pp.639-644,2009.DOI:https://doi.org/ 10.1016/j.resconrec.2009.04.014.

[4] M. Asif, T. Muneer y R. Kelley, "Life cycle assessment: A case study of a dwelling home in Scotland," Building and Environment, vol. 42, n.| 3, pp. 1391-1394, 2007. DOI: https://doi. org/10.1016/j.buildenv.2005.11.023.

[5] O. Yepes y C. Bedoya, "El bloque de suelo cemento (BSC) al bloque de suelo," tesis de Maestría en Construcción, Modalidad Profundización, énfasis en Construcción Sostenible, Universidad Nacional de Colombia Sede Medellín, Medellín, Colombia, 2012. [En línea]. Disponible en: http://www.bdigital unal. edu.co/8561/1/43635688.2012.pdf. [Último acceso: 2 Junio 2017]. 
[6] J. Hernandez, "El impacto ambiental por la actividad de explotación de canteras en la localidad de Usme y sus principales medidas de manejo," tesis, Especialización en Planeación Ambiental y Manejo Integral de los Recursos $\mathrm{Na}$ turales, Universidad Militar Nueva Granada, Bogotá, Colombia 2015. [En línea]. Disponible en: https: / / repository.unimilitar.edu.co/bitstre am/handle/10654/6331/Art\%EDculo\%20EL; jsessionid=E0023147ED6C0A8A232A9547860A DF53? sequence $=1$.

[7] S. Roh, S. Taeb, S. Joon y G. Fordc, "Evaluating the embodied environmental impacts of major building tasks and materials of apartment buildings in Korea," Renewable and Sustainable Energy Reviews, vol. 73, pp. 135-144, 2017. DOI: https://doi.org/10.1016/j.rser.2017.01.081.

[8] H. Yan, Q. Shen, L. Fan, Y. Wamg y L. Zhang, "Greenhouse gas emissions in building construction: A case study of One Peking in Hong Kong," Building and Environment, vol. 45, n. ${ }^{\circ}$ 4, pp. 949-955, abr., 2010. DOI: 10.1016/ j.buildenv.2009.09.014

[9] O. Ortiz, F. Castells y G. Sonnemann, "Sustainability in the construction industry: A review of recent developments based on LCA," Construction and Building Materials, vol. 23, n. 1, pp. 28-39, 2009. DOI: https://doi. org/10.1016/j.conbuildmat.2007.11.012

[10] H. J. Wu, Z. W. Yuan, L. Zhang y J. Bi, "Life cycle energy consumption and $\mathrm{CO} 2$ emission of an office building in China," Building and Environment, vol. 17, n. ${ }^{\circ}$ 2, pp. 36-48, 2012. [En línea]. Disponible en https: / /link.springer. com/article/10.1007/s11367-011-0342-2.

[11] O. Ortiz, F. Castells y G. Sonnemann, "Life cycle assessment of two dwellings: One in Spain, a developed country, and one in Colombia, a country under development," Science of The Total Environment, vol. 408, n. ${ }^{\circ}$ 12, pp. 2435-2443,2010. DOI: https://doi. org/10.1016/j.scitotenv.2010.02.021.

[12] A. V. Cuevas, "Estado del arte sobre el Análisis de Ciclo de Vida en la construccion de vivienda potencial de aplicación en Colombia,"trabajo de grado, Administración y Grestión Ambiental, Universidad Piloto de Colombia, Bogotá, 2012. Disponible en: http: / / polux.unipiloto.edu.co: 8080/00000438.pdf
[13] M. Glavind y C. Munich-Petersen, “'Green' Concrete in Denmark," Structural Concrete, n. ${ }^{\circ}$, pp. 19-25, 2000. [En línea]. Disponibleen: https://www.dti.dk/green-concrete-in-den mark/2496.

[14] A. C. d. C. Filho, "Análisis del ciclo de vida de productos derivados del cemento - Aportaciones al análisis de los inventarios del ciclo de vida del cemento," Universidad Politécnica de Cataluña, Barcelona, España, 2001. [En línea]. Disponible en: http://hdl.handle. net $/ 10803 / 5903$

[15] K. Onabolu y R. T, "A case study of the development of life cycle assessment as a management tool in a building industry organisation," en International Symposium on Intergrated Life-Cycle Design of Materials and Structures, Helsinki, Finlandia, 2000.

[16] M. Vold y A. Ronning, "LCA of cement and concrete - Main report," Finncement $A B, \mathrm{Ce}$ menta $A B$ and Norcem AS, Fredrikstad, Noruega, nov., 1995 pp. 50-50, 1995. [En línea]. Disponible en: https: //www.ostfoldforskning. no/media/1503/3295.pdf.

[17] M. Nisbet y M. Van Geem, “Environmental Life Cycle Inventory of Portland Cement and Concrete," World Cement, 1997. [En línea]. Disponible en: https://www.nrmca.org/taskforce/Item_2_TalkingPoints/Sustainability/ Sustainability/SN2140.pdf.

[18] C. Muñoz y F. Quiroz, "Análisis de Ciclo de Vida en la determinación de energía contenida y la huella de carbono en el proceso de fabricación del hormigón premezclado. Caso estudio planta productora Región del Bio Bío, Chile," Revista Hábitat Sustentable, vol. 4, n. 2, pp. 16-25, 2014. [En línea]. Disponible en: http://revistas.ubiobio.cl/index.php/ $\mathrm{RHS} /$ article/view/447.

[19] L. P. Rosado, P. Vitale, C. Santos y H. Arena, "Life cycle assessment of natural and mixed recycled aggregate production in Brazil," Journal of Cleaner Production, vol. 151, pp. 634-642, 2017. DOI: https://doi.org/10.1016/j.jclepro.2017.03.068.

[20] Agencia Suiza para el Desarrollo y la Cooperación y la Pontificia Universidad Católica del Perú, Estudio de análisis de ciclo de vida de ladrillos y bloques de concreto, Lima: Pontificia Universidad Católica del Perú, 2010. 
[21] L. Zhang, "Production of bricks from waste materials-Areview," Constructionand Building Materials, vol. 47, pp. 643-655, 2013. DOI: https://doi.org/10.1016/j.conbuildmat. 2013.05.043.

[22] I. SartoriyA. Hestnes, "Energy use in the life cycle of conventional and low-energy buildings: a review article," Energy and Buildings, vol. 39, n. ${ }^{\circ}$ 3, pp. 249-257, 2007. DOI: https://doi. org/10.1016/j.enbuild.2006.07.001

[23] K. Adalberth, A. Almgren y E. Petersen, "Life cycle assessment of four multi-family buildings, "International Journal of LowEnergyand Sustainable Buildings, vol. 2, pp. 1-21, 2001.

[24] B. Peuportier, "Life cycle assessment applied to the comparative evaluation of single family houses in the French context," Energy and Buildings, vol. 33, n. ${ }^{\circ}$ 5, pp. 443-450, 2001. DOI: https://doi.org/10.1016/S03787788(00)00101-8

[25] F. Asdrubalia, C. Baldassarria y V. Fthenakisb, "Life cycle analysis in the construction sector: Guiding the optimization of conventional Italian buildings," Energy and Buildings, vol. 64, pp. 73-89, 2013. DOl: https://doi. org/10.1016/j.enbuild.2013.04.018.

[26] P. Vitale, N. Arenab, F. Di Gregorioc y U. Arenaa, "Lifecycleassessment of theend-of-lifephase of a residential building," Waste Management, vol. 60, p. 311-321, 2017. DOI:10.1016/j.wasman. 2016.10.002.

[27] G. A. Blengini, “ Life cycle of buildings, demolition and recycling potential: a casestudy in Turin, Italy. " Build Environ, vol 44, pp. 319330, 2009. DOI: https://doi.org/10.1016/j. buildenv.2008.03.007

[28] F. Ardente, G. Beccali, M. Cellura, M. Fontana y S. Longo, "L'analisi del ciclo di vita applicata agli edifici residenziali: il caso studio di un edificio mono-familiare, "Rivista La Termotécnica, n. ${ }^{\circ} 7$, pp. 55-59, 2009.

[29] L. Huang, Y. Liu, G. Krigsvoll y F. Johansen, "Life cycle assessment and life cycle cost of university dormitories in the southeast China: Case study of the university town of Fuzhou," Journal of Cleaner Production, vol. 173, pp. 151-159, 2018. DOI: https://doi.org/10.1016/j.jclepro.2017.06.021.

[30] M. Sandanayakea, Guomin, S. Setungea, W. Luob, Lia y Chun-Qing, "Estimation and comparison of environmental emissions and impacts at foundation and structure construction stages of a building - A case study," Journal of Cleaner
Production, vol. 151, pp. 319-329, 2017. DOI: https://doi.org/10.1016/j.jclepro.2017.03.041.

[31] C. Scheuer, G. A. Keoleian y P. Reppe, "Life cycle energy and environmental performance of a new university building: modeling challenges and design implications," Energy and Buildings, vol. 35, n. ${ }^{\circ}$ 10, pp. 1049-1064, 2003. DOI: https://doi.org/10.1016/S03787788(03)00066-5

[32] ISO 14040, “Gestión Ambiental - Análisis de Ciclo de Vida - Principios y Marco de referencia," Instituto Colombiano de Normas Técnicas y Certificación ICONTEC, 2007.

[33] EPM, “Empresa de servicios públicos de Medellín y Colombia," [En línea]. Disponible en: http://www.epm.com.co/site/. [último acceso: 2 Marzo 2017].

[34] Ecoinvent, “Ecoinvent 3.3,” 2016.[En línea]. Disponible en: https: / /www.ecoinvent.org/

[35] M. Tait y W. Cheung, "AComparative Cradle-toGate Life Cycle Assessment of Three Concrete Mix Designs," The International Journal of LifeCycle Assessment, vol. 21, n. ${ }^{\circ}$ 6, pp. 847860, 2016. [En línea]. Disponible en: https:// link.springer.com/article/10.1007/s11367016-1045-5.

[36] C. Knoeri, E. Sanyé-Mengual y H.-J. Althaus, "Comparative LCA of recycled and conventional concrete for structural applications," The International Journal of Life Cycle Assessment, vol. 18, n. ${ }^{\circ}$ 5, pp. 909-918, 2013. [En línea]. Disponible en https://link.springer. com/article/10.1007/s11367-012-0544-2

[37] C. Dossche, V. Boel, W. De Corte y N. De Belie, "Green concrete: optimization of highstrength concrete based on LCA» Durability and Sustainability of Concrete Structures, Workshop Proceedings, Michigan, American Concrete Institute, 2015, pp. 357-366.

[38] Instituto de Ciencias Medioambientales, "CML," Universidad de Leiden en los Países Bajos, Holanda, 2001.

[39] Open LCA, “Open LCA 1.6.3,” 2017. http:// www.openlca.org/

[40] N. Lee, S. Tae, Y. Gong y S. Roh, "Integrated building life-cycle assessment model to support South Korea's green building certification system (G-SEED)," Renewable and Sustainable Energy Reviews, vol. 76, pp. 43-50, 2017. DOI: https://doi.org/10.1016/j.rser.2017.03.038. 
[41] Dirección de Gestión Ambiental y Ecosistemas, 2010.Guía para el manejo ambiental de obras.

[42] Secretaría del Medio Ambiente de Medellín, Empresas Públicas de Medellín, Manual de Gestión Socio-Ambiental para Obras de Construcción, 2009.

[43] A. Hess, "Impacto Ambiental de Componentes y Materiales de Edificios," 2005. [Internet]. Disponibleen:https://studylib.es/doc/5107761 /impacto-ambiental-de-componentesy-materiales-de-edificios.

[44] J. Muro y J. Fernandez, "Análisis de ciclo de vida de $1 \mathrm{kWh}$ generado por un parque eólico," Gamesa Corporación Tecnológica, España, 2013.

[45] A. Latorre, "La industria del cemento en Colombia. Determinantes y comportamiento de la demanda,", trabajo de grado pregrado, Facultad de Ciencias Económicas y Administrativas, Pontificia Universidad Javeriana, Bogotá, 2008.

[46] B. Estanqueiro, J. Silvestre, Brito, Jorge y M. Duarte, "Environmental life cycle assessment of coarse natural and recycled aggregates for concrete," European Journal of Environmental and Civil Engineering, vol. 22, n. ${ }^{\circ} 4$, pp. 1-21, 2016. DOI: https://doi.org/1 0.1080/19648189.2016.1197161.

[47] C. Bories, E. Vedrenne, A. P. Massol, G. Vilarem y C. Sablayrolles, "Development of porous fired clay bricks with bio-based additives: Study of the environmental impacts by Life Cycle Assessment (LCA)," Construction and Building Materials, vol. 125, pp. 11421151, 2016. DOI: https://doi.org/10.1016/j. conbuildmat.2016.08.042.

[48] M. Valverde Vera, E. Bances Zapata, A. Rojas y B. Rodriguez, "Impactos ambiental producido por la fabricación de ladrillos en el valle de alto mayo - San Martín," tesis, Programa Ingeniería Ambiental, Universidad Nacional de San Martín, Perú, 2004.

[49] M. Gousi, C. Andriopoulou, K. Bourikas, S. Ladas, M. Sotiriou, C. Kordulis y A.
Lycourghiotis, "Green diesel production over nickel-alumina co-precipitated catalysts," Applied Catalysis A: General, vol. 536, pp. 45-56, 2017. DOI: https: //doi.org/10.1016/j. apcta.2017.02.010.

[50] B. Boonrod, C. Prapainainar, P. Narataruksa, A. Kantama, W. Saibautrong, K. Sudsakorn, T. Mungcharoen y P. Prapainainar, "Evaluating the environmental impacts of bio-hydrogenated diesel production from palm oil and fatty acid methyl ester through life cycle assessment," Journal of Cleaner Production, vol. 142, parte 3, pp. 1210-1221, 2017. DOI: https://doi.org/10.1016/j.jclepro.2016.07.128.

[51] M. Kucukvar, M. Ali Haider y N. CihatOnat, "Exploring the material footprints of national electricity production scenarios until 2050: The case for Turkey and UK," Resources, Conservation and Recycling, vol. 125, pp. 251263, 2017. DOI: https://doi.org/10.1016/j. resconrec.2017.06.024.

[52] M. A. Shaikha, M. Kucukvara, N. CihatOnata y G. Kirkil, "A framework for water and carbon footprint analysis of national electricity production scenarios," Energy, vol. 139, pp. 406-421, 2017. DOI: https://doi. org/10.1016/j.energy.2017.07.124.

[53] Electrificadora del Caribe S.A, “Generación, distribución y medición," [En línea]. Disponible en http://www.electricaribe.com/co/ grandes+clientes/distribucion+de+electri cidad/1297110325183/generacion+distribu cion+y+medicion.html. [Último acceso: 1 Septiembre 2017].

[54] Directiva 2008/98/CE, "Sobre residuos y por la que se derogan determinadas Directivas," Parlamento Europeo y del Consejo, 2008.

[55] T. Ramesh, R. Prakash y K. Shukla, "Life cycle energy analysis of buildings: An overview," Energy and Buildings, vol. 42, $\mathrm{n}{ }^{\circ}$ 10, pp. 1592-1600, 2010. DOI: https://doi. org/10.1016/j.enbuild.2010.05.007. 